\title{
Nonlinear theory of wakefield excitation in a rectangular multizone dielectric resonator
}

\author{
K. V. Galaydych ${ }^{1}$ and G. V. Sotnikov ${ }^{1,2}$ \\ ${ }^{1}$ NSC Kharkov Institute of Physics and Technology, 1 Akademicheskaya Street, Kharkov 61108, Ukraine \\ ${ }^{2}$ Omega-P, Inc., 258 Bradley Street, New Haven, Connecticut 06510, USA
}

(Received 21 October 2010; published 31 January 2011)

\begin{abstract}
A nonlinear self-consistent theory has been constructed and used to investigate numerically the wakefield excitation in multilayered dielectric resonators by relativistic electron bunches. Analytical expressions for solenoidal and potential components of an excited electromagnetic field have been derived. The excitation of a five-zone dielectric resonator by relativistic electron bunches was numerically investigated and comparison was made between the longitudinal distribution of an axial electric field and the results obtained previously for a corresponding problem in the waveguide formulation. The necessity of optimizing geometrical parameters of the resonator to reduce mode amplitudes nonresonant with a bunch, and to obtain a symmetric distribution of the longitudinal electric field component in the drive and accelerating channels, has been demonstrated.
\end{abstract}

DOI: 10.1103/PhysRevSTAB.14.011302

PACS numbers: 41.75.Lx, 41.75.Ht, 96.50.Pw, 41.75.Jv

\section{INTRODUCTION}

The elaboration of waveguide dielectric structures for the problems of charged particle accelerator physics is an actively developing direction in both theoretical and experimental studies. The review reports presented at the recent conferences [1,2], and also, the reports concerned with the projects of future colliders [3] have demonstrated the progress and prospects for the investigations carried out along with traditional methods of acceleration.

A promising method of charged particle acceleration is the two-beam acceleration by wakefields excited by charged particle bunches in dielectric structures. The two-beam methods of acceleration are based on the following principle: highly charged, relativistic bunches generate an intense longitudinal field whereby the test bunches are accelerated. Experimental evidence of the given principle of charged particle acceleration has been clearly demonstrated in the studies [4] carried out at ANL. It is well known [5-7] that the transformer ratio, which specifies the maximum energy of accelerated particles, can be no more than 2 if the exciting and accelerated bunches traverse the structure in the same path. To overcome this restriction, it is possible to profile a bunch train [8]. The other way is to use multilayered dielectric structures with spaced vacuum channels for the exciting and accelerated bunches [9]. In the multizone accelerating structure the transform ratio can be controlled by varying the drive and accelerating channel widths [10]. In the multizone structure it is also possible to make the mode having a symmetrical distribution of axial electric field in vacuum channels act as an operating mode. This additional advantage of multizone structures allows one to diminish transverse deflecting forces.

The previous theoretical investigations on wakefield excitation in multizone dielectric structures have been carried out for longitudinally unbounded structures [10]. Practicable accelerating structures are bounded in the travel direction of exciting and accelerated bunches. Therefore the effects associated with transition radiation and the group velocity of excited waves [11] take place in the structures. The results of numerical simulations based on the particle-in-cell (PIC) method show that the effects associated with longitudinal boundedness of the accelerating structure cannot be neglected, especially with advancement to the $\mathrm{THz}$ frequency range $[10,12]$.

The effects related to the group velocity of an excited wakefield and the transition radiation can be taken into account most simply in the resonator formulation of the problem [13]. In the present paper we investigate these effects using as an example the excitation of a five-zone rectangular dielectric structure by electron bunches [10]. For this purpose, in Sec. II (statement of the problem) and in Sec. III we form the general nonlinear theory of excitation of multizone rectangular dielectric resonators, valid for any number of dielectric layers [14]. Besides taking account of the group velocity effects, this theory allows one to consider a self-consistent influence of bunch dynamics on the excited wakefields. Previously, to take into account this dynamics, fully numerical methods $[10,12]$ have been used. However, their applicability is often limited by current computer capacities. This problem is topical when simulating wakefield excitation of the $\mathrm{THz}$ frequency range. Here we offer a combined method of considering the influence of bunch dynamics on the wakefield excitation. Expressions for the excited fields are derived by analytical methods. They are functionally dependent on particle location at any time point (a nonlinear Green's function). Together with the equations of motion they provide a self-consistent description of the dynamics of fields and bunches.

In Sec. IV the theory developed is applied to calculate numerically the wakefield excitation in the five-zone dielectric resonator. The calculated data are compared with the 
results of the linear theory of excitation of the five-zone dielectric structure, obtained in waveguide formulation [10].

\section{STATEMENT OF THE PROBLEM}

The multizone dielectric structure under investigation is a rectangular metal resonator with dielectric slabs placed in parallel to one of the walls [15]. We shall direct the $z$ axis of the Cartesian coordinate system in parallel to the direction of the beams, and both the $x$ and $y$ axes are transverse to the beam direction with the $x$ axis perpendicular to the slabs. The dielectric slabs generally have different values of a permittivity $\varepsilon=\varepsilon(x)=\varepsilon_{i}(1 \leq i \leq N)$ and permeability $\mu=\mu(x)=\mu_{i}(1 \leq i \leq N) ; N$ is the number of zones of the dielectric structure. In one of the vacuum zones $(\varepsilon=1$, $\mu=1$ ), the electron bunch is injected into the resonator at $z=0 . a, b$, and $L$ denote the width, height, and length of the resonator, respectively. We suppose that the end walls of the resonator are closed by metal grids transparent for charged particles and nontransparent for an excited electromagnetic field. The cross section of the multilayered dielectric resonator together with the chosen system of coordinates is schematically shown in Fig. 1. The same figure shows the exciting bunch position in one of the vacuum zones. For ease of further numerical calculations (Sec. IV), we consider the bunch to be rectangular in shape.

Let us express the required fields as a sum of solenoidal and potential components [16]:

$$
\mathbf{E}=\mathbf{E}^{\mathbf{t}}+\mathbf{E}^{l}, \quad \mathbf{H}=\mathbf{H}^{\mathbf{t}},
$$

where $\mathbf{E}^{\mathbf{t}}$ and $\mathbf{H}^{\mathbf{t}}$, being the solenoidal components of the electromagnetic field $\left(\operatorname{div}\left(\varepsilon \mathbf{E}^{\mathbf{t}}\right)=0, \operatorname{div}\left(\mu \mathbf{H}^{\mathbf{t}}\right)=0\right)$, satisfy the first and the second Maxwell equations,

$$
\begin{aligned}
& \nabla \times \mathbf{H}^{\mathbf{t}}=\frac{\varepsilon(x)}{c} \frac{\partial \mathbf{E}^{\mathbf{t}}}{\partial t}+\frac{4 \pi}{c} \mathbf{j}, \\
& \nabla \times \mathbf{E}^{\mathbf{t}}=-\frac{\mu(x)}{c} \frac{\partial \mathbf{H}^{\mathbf{t}}}{\partial t},
\end{aligned}
$$

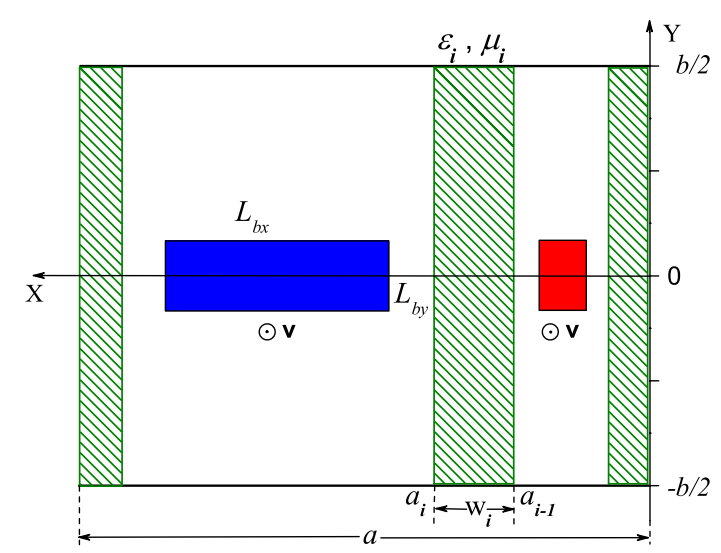

FIG. 1. Cross section of the five-zone resonator. The blue rectangle shows the drive bunch, the red rectangle shows the test bunch, and a small circle with a central dot and the bunch velocity vector indicate that the beam motion is out of the page. and the potential electric field $\mathbf{E}^{l}\left(\nabla \times \mathbf{E}^{l}=0\right)$ satisfies the Gauss law,

$$
\operatorname{div}\left(\varepsilon \mathbf{E}^{l}\right)=4 \pi \rho .
$$

The solenoidal $\mathbf{E}^{\mathbf{t}}$ and potential $\mathbf{E}^{l}$ electric fields are mutually orthogonal [16] and satisfy the boundary conditions, making their tangential components vanish on the metal walls of the resonator:

$$
\mathbf{E}_{\tau}^{l}\left(\mathbf{r} \in S_{0}\right)=0, \quad \mathbf{E}_{\tau}^{\mathbf{t}}\left(\mathbf{r} \in S_{0}\right)=0,
$$

where $S_{0}$ designates the metal surface of the resonator, and the subscript $\tau$ denotes the tangential component of the fields.

The electron bunches will be described in terms of macroparticles, therefore the charge density $\rho$ and the current density $\mathbf{j}$ will be written as

$$
\begin{aligned}
\rho & =\sum_{p \in V_{R}} q_{p} \delta\left[\mathbf{r}-\mathbf{r}_{p}(t)\right], \\
\mathbf{j} & =\sum_{p \in V_{R}} q_{p} \mathbf{v}_{p}(t) \delta\left[\mathbf{r}-\mathbf{r}_{p}(t)\right],
\end{aligned}
$$

where $q_{p}$ is the charge of the macroparticle, $\mathbf{r}_{p}$ and $\mathbf{v}_{p}$ are its time-dependent coordinates and velocity, respectively. The summation in Eq. (5) is carried out over the particles being in the resonator volume $V_{R}$.

The self-consistent dynamics of bunch particles is described by relativistic equations of motion in the electromagnetic fields excited by bunches:

$$
\frac{d \mathbf{p}_{p}}{d t}=q_{p}\left(\mathbf{E}+\frac{1}{m_{p} c \gamma_{p}} \mathbf{p}_{p} \times \mathbf{B}\right), \quad \frac{d \mathbf{r}_{p}}{d t}=\frac{\mathbf{p}_{p}}{m_{p} \gamma_{p}},
$$

where $\gamma_{p}^{2}=1+\left(\mathbf{p}_{p} / m_{p} c\right)^{2}$.

\section{GREEN'S FUNCTION OF THE PROBLEM}

Let us derive analytical solutions to the set of Eqs. (1)-(5) that will allow us to avoid their numerical solution on a spatial grid. Therein lies the essential difference from numerical algorithms obtained with the use of the PIC method. We first find the potential electric field, which can be represented as $\mathbf{E}^{l}=-\nabla \Phi$. Its finding is reduced to solving the Poisson equation,

$$
\frac{1}{\varepsilon(x)} \frac{\partial}{\partial x}\left(\varepsilon(x) \frac{\partial \Phi}{\partial x}\right)+\frac{\partial^{2} \Phi}{\partial y^{2}}+\frac{\partial^{2} \Phi}{\partial z^{2}}=-\frac{4 \pi}{\varepsilon} \rho,
$$

together with the boundary conditions consisting in that the potential $\Phi$ on the resonator metal walls becomes zero:

$$
\begin{aligned}
\Phi(x & =0)=\Phi(x=a)=\Phi(x= \pm b / 2)=\Phi(z=0) \\
& =\Phi(z=L)=0
\end{aligned}
$$


and it is continuous at the zone boundaries

$$
\Phi\left(x=a_{i}-0\right)=\Phi\left(x=a_{i}+0\right) .
$$

Equation (7) will be solved by the eigenfunction expansion method. The eigenvalues and eigenfunctions can be found from the solution of the Sturm-Liouville problem,

$$
\frac{1}{\varepsilon(x)} \frac{\partial}{\partial x}\left(\varepsilon(x) \frac{\partial \Phi_{s}}{\partial x}\right)+\frac{\partial^{2} \Phi_{s}}{\partial y^{2}}+\frac{\partial^{2} \Phi_{s}}{\partial z^{2}}+\lambda_{s} \Phi_{s}=0,
$$

where $\lambda_{s}$ is the eigenvalue, and $\Phi_{s}$ is the corresponding eigenfunction.

Let us solve Eq. (10) by the Fourier method. Representing the solutions in the form

$$
\Phi_{s}(x, y, z)=X_{m}(x) Y_{n}(y) Z_{l}(z)
$$

and taking into account the boundary conditions, we obtain for $Y_{n}(y)$ and $Z_{l}(z)$ :

$$
Y_{n}(y)=\sqrt{\frac{2}{b}} \sin k_{y}^{n}\left(y+\frac{b}{2}\right), \quad Z_{l}(z)=\sqrt{\frac{2}{L}} \sin k_{z}^{l} z,
$$

where $k_{y}^{n}=\frac{\pi n}{b}, k_{z}^{l}=\frac{\pi l}{L}$.

The functions $X_{m}(x)$, which define the dependence of $\Phi_{s}$ on the transverse $x$ coordinate, are the solutions of the equation

$$
\frac{d}{d x}\left(\varepsilon(x) \frac{d X_{m}}{d x}\right)+k_{x}^{2} X_{m}(x)=0
$$

with unknown values of $k_{x}^{2}$.

For deriving the solution of Eq. (13), it is reasonable to use the matrix method of finding eigenfunctions in a multizone waveguide [15]. Taking into account the boundary conditions consisting in the continuity of both the potential (9) and the transverse component of electric induction vector

$$
\left.\varepsilon_{i} \frac{d X_{m}}{d x}\right|_{x=a_{i}-0}=\left.\varepsilon_{i+1} \frac{d X_{m}}{d x}\right|_{x=a_{i}+0},
$$

for the functions $\varphi^{(i)}(x) \equiv X_{m}\left(a_{i-1} \leq x \leq a_{i}\right)$ we obtain the following expression:

$$
\begin{aligned}
\varphi_{m}^{(i)}(x) & =\left(\cos k_{x}^{m}\left(x-a_{i}\right), \frac{1}{\varepsilon_{i} k_{x}} \sin k_{x}^{m}\left(x-a_{i}\right)\right) \chi_{m}^{(i)}, \\
\chi_{m}^{(i \leq N-2)} & =\left(\prod_{j=i+1}^{N-1 \geq 2} V^{(j)}\right)\left(\begin{array}{c}
-\sin k_{x} w_{N} \\
\varepsilon_{N} k_{x} \cos k_{x} w_{N}
\end{array}\right), \\
\chi_{m}^{(N-1)} & =\left(\begin{array}{c}
-\sin k_{x} w_{N} \\
\varepsilon_{N} k_{x} \cos k_{x} w_{N}
\end{array}\right), \\
\chi_{m}^{(N)} & =\left(\begin{array}{c}
0 \\
\varepsilon_{N} k_{x}
\end{array}\right), \\
V^{(i)} & =\left(\begin{array}{cc}
\cos k_{x} w_{i} & -\frac{\sin k_{x} w_{i}}{\varepsilon_{i} k_{x}} \\
\varepsilon_{i} k_{x} \sin k_{x} w_{i} & \cos k_{x} w_{i}
\end{array}\right),
\end{aligned}
$$

where $i$ is the zone number $(1 \leq i \leq N), w_{i}=a_{i}-a_{i-1}$ and $\varepsilon\left(a_{i-1} \leq x \leq a_{i}\right) \equiv \varepsilon_{i}$ are the width and permittivity of the $i$ th zone, correspondingly; $a_{i}$ is the right coordinate of the $i$ th zone, $a_{0}=0, a_{N}=a$.

From Eq. (13) and boundary conditions (9) and (14) the orthogonality conditions of the eigenfunctions $X_{m}(x)$ and their norm $\left\|X_{m}\right\|^{2}$ result:

$$
\begin{aligned}
& \frac{2}{a} \int_{0}^{a} d x \varepsilon(x) X_{m}(x) X_{m^{\prime}}(x)=\left\|X_{m}\right\|^{2} \delta_{m m^{\prime}}, \\
& \left\|X_{m}\right\|^{2}=\frac{2}{a} \sum_{i=1}^{N} \varepsilon_{i} \int_{a_{i-1}}^{a_{i}} d x\left[\varphi_{m}^{(i)}(x)\right]^{2} .
\end{aligned}
$$

The $k_{x}$ eigenvalues are defined from the dispersion equation:

$$
\left(\cos k_{x} w_{1},-\frac{\sin k_{x} w_{1}}{\varepsilon_{1} k_{x}}\right)\left(\prod_{j=2}^{N-1 \geq 2} V^{(j)}\right)\left(\begin{array}{c}
-\sin k_{x} w_{N} \\
\varepsilon_{N} k_{x} \cos k_{x} w_{N}
\end{array}\right)=0 .
$$

Thus, the final expression for the potential $\Phi$ can be written as

$$
\begin{aligned}
\Phi= & \frac{32 \pi}{a b L} \sum_{p, m n l} \frac{q_{p} X_{m}\left(x_{p}(t)\right) \sin k_{y}^{n}\left(y_{p}(t)+b / 2\right) \sin k_{z}^{l} z_{p}(t)}{\left\|X_{m}\right\|^{2}\left[\left(k_{x}^{m}\right)^{2}+\left(k_{y}^{n}\right)^{2}+\left(k_{z}^{l}\right)^{2}\right]} \\
& \times X_{m}(x) \sin k_{y}^{n}(y+b / 2) \sin k_{z}^{l} z .
\end{aligned}
$$

The solenoidal fields $\mathbf{E}^{\mathbf{t}}$ and $\mathbf{H}^{\mathbf{t}}$ satisfy the equations

$\nabla \times \mathbf{H}^{\mathbf{t}}=\frac{\varepsilon(x)}{c} \frac{\partial \mathbf{E}}{\partial t}+\frac{4 \pi}{c} \mathbf{j}, \quad \nabla \times \mathbf{E}^{\mathbf{t}}=-\frac{\mu(x)}{c} \frac{\partial \mathbf{H}^{\mathbf{t}}}{\partial t}$.

The solenoidal parts of the bunch-excited electromagnetic field can be determined by expanding the required fields into solenoidal fields of the empty multizone resonator. Let us write down the fields $\mathbf{E}^{\mathbf{t}}$ and $\mathbf{H}^{\mathbf{t}}$ in the form

$$
\mathbf{E}^{\mathbf{t}}=\sum_{s} A_{s}(t) \mathbf{E}_{s}(\mathbf{r}), \quad \mathbf{H}^{\mathbf{t}}=-i \sum_{s} B_{s}(t) \mathbf{H}_{s}(\mathbf{r}) .
$$

The functions $\mathbf{E}_{s}$ and $\mathbf{H}_{s}$, which describe the spatial structure of solenoidal fields, satisfy the Maxwell sourcesfree equations

$$
\nabla \times \mathbf{H}_{s}=-i k_{s} \varepsilon(x) \mathbf{E}_{s}, \quad \nabla \times \mathbf{E}_{s}=i k_{s} \mu(x) \mathbf{H}_{s},
$$

where $k_{s}=\omega_{s} / c, \omega_{s} \equiv \omega_{m n l}$ are the eigenfrequencies of the dielectric resonator.

Using the orthogonality conditions of $\mathbf{E}_{s}$ and $\mathbf{H}_{s}$ [16],

$$
\int_{V_{R}} d V \varepsilon \mathbf{E}_{s} \mathbf{E}_{s^{\prime}}^{*}=\int_{V_{R}} d V \mu \mathbf{H}_{s} \mathbf{H}_{s^{\prime}}^{*}=4 \pi N_{s} \delta_{s s^{\prime}}
$$

for finding the field amplitudes $A_{s}(t)$ and $B_{s}(t)$, we obtain the following nonuniform second-order differential equations: 


$$
\begin{aligned}
& \frac{d^{2} A_{s}}{d t^{2}}+\omega_{s}^{2} A_{s}=-\frac{d R_{s}}{d t}, \\
& \frac{d^{2} B_{s}}{d t^{2}}+\omega_{s}^{2} B_{s}=-\omega_{s} R_{s}, \\
& R_{s}(t)=\frac{1}{N_{s}} \sum_{p \in V_{R}} q_{p} \mathbf{v}_{p}(t) \mathbf{E}_{s}^{*}\left[\mathbf{r}=\mathbf{r}_{p}\left(t, t_{0}\right)\right] .
\end{aligned}
$$

Having solved Eq. (24) we have

$$
\begin{aligned}
& B_{s}(t)=-\int_{0}^{t} d t^{\prime} \sin \omega_{s}\left(t-t^{\prime}\right) R_{s}\left(t^{\prime}\right), \\
& A_{s}(t)=\frac{1}{\omega_{s}} \frac{d B_{s}}{d t} .
\end{aligned}
$$

In the case, where the permittivity and permeability are nonuniform only along the direction normal to the slabs, the electromagnetic-field solenoidal components in multizone dielectric structures can be represented as a superposition of longitudinal section magnetic (LSM) and longitudinal section electric (LSE) waves $[17,18]$. In the LSM wave, the magnetic field component $H_{x s}$, which is transverse to the dielectric slabs, is equal to zero. The other components of the LSM waves are expressed in terms of the transverse electric field component $E_{x s}$. In the LSE wave, the electric field component $E_{x s}$, which is transverse to the dielectric slabs, is equal to zero, and the other five components of the LSE waves can be obtained using the transverse magnetic field component $H_{x s}$. The field components of LSM and LSE waves, which have been obtained in $[15,19]$ for the waveguide case, are generalized for the resonator structure under study by replacing the continuous longitudinal wave number $k_{z}$ with its discrete values $k_{z}^{l}=\pi l / L$ in the resonator.

The field components of LSM waves are given by

$$
\begin{aligned}
H_{x s} & =0, \\
H_{y s} & =\frac{-i k_{s}}{\kappa_{n l}^{2}} \frac{\partial}{\partial z}\left(\varepsilon E_{x s}\right), \\
H_{z s} & =\frac{i k_{s}}{\kappa_{n l}^{2}} \frac{\partial}{\partial y}\left(\varepsilon E_{x s}\right), \\
E_{y s} & =\frac{1}{\kappa_{n l}^{2} \varepsilon} \frac{\partial^{2}}{\partial x \partial y}\left(\varepsilon E_{x s}\right), \\
E_{z s} & =\frac{1}{\kappa_{n l}^{2} \varepsilon} \frac{\partial^{2}}{\partial x \partial z}\left(\varepsilon E_{x s}\right), \\
E_{x s} & =e_{x s}^{(i)}(x) \sin k_{y}^{n}(y+b / 2) \sin k_{z}^{l} z,
\end{aligned}
$$

where $\kappa_{n l}^{2}=\left(k_{y}^{n}\right)^{2}+\left(k_{z}^{l}\right)^{2}$, and the functions $e_{x s}^{(i)}(x)$ are defined as

$$
e_{x s}^{(i)}=\frac{1}{\varepsilon_{i}}\left(\cos k_{x s}^{i}\left(a_{i}-x\right),-\frac{\varepsilon_{i}}{k_{x s}^{i}} \sin k_{x s}^{i}\left(a_{i}-x\right)\right) \xi_{s}^{(i)},
$$

$$
\begin{aligned}
\xi_{s}^{(i \leq N-2)} & =\left(\prod_{j=i+1}^{N-1} S_{s}^{(j)}\right)\left(\begin{array}{c}
\cos k_{x s}^{N} w_{N} \\
\frac{k_{x s}^{N}}{\varepsilon_{N}} \sin k_{x s}^{N} w_{N}
\end{array}\right) \frac{\varepsilon_{N}}{\cos k_{x s}^{N} a_{N}}, \\
\xi_{s}^{(N-1)} & =\left(\begin{array}{c}
\cos k_{x s}^{N} w_{N} \\
\frac{k_{x s}^{N}}{\varepsilon_{N}} \sin k_{x s}^{N} w_{N}
\end{array}\right) \frac{\varepsilon_{N}}{\cos k_{x s}^{N} a_{N}}, \\
\xi_{s}^{(N)} & =\left(\begin{array}{c}
1 \\
0
\end{array}\right) \frac{\varepsilon_{N}}{\cos k_{x s}^{N} a_{N}}, \\
S^{(i)} & \equiv\left(\begin{array}{cc}
\cos k_{x}^{i} w_{i} & -\frac{\varepsilon_{i}}{k_{x}^{i}} \sin k_{x}^{i} w_{i} \\
\frac{k_{x}^{i}}{\varepsilon_{i}} \sin k_{x}^{i} w_{i} & \cos k_{x}^{i} w_{i}
\end{array}\right),
\end{aligned}
$$

where $\left(k_{x}^{i}\right)^{2}=\omega^{2} \varepsilon_{i} \mu_{i} / c^{2}-\left(k_{y}^{n}\right)^{2}-\left(k_{z}^{l}\right)^{2}$.

For the LSE waves, the field components are written down as

$$
\begin{aligned}
E_{x s} & =0, \\
E_{y s} & =\frac{i k_{s}}{\kappa_{n l}^{2}} \frac{\partial}{\partial z}\left(\mu H_{x s}\right), \\
E_{z s} & =\frac{-i k_{s}}{\kappa_{n l}^{2}} \frac{\partial}{\partial y}\left(\mu H_{x s}\right), \\
H_{y s} & =\frac{1}{\kappa_{n l}^{2} \mu} \frac{\partial^{2}}{\partial x \partial y}\left(\mu H_{x s}\right), \\
H_{z s} & =\frac{1}{\kappa_{n l}^{2} \mu} \frac{\partial^{2}}{\partial x \partial z}\left(\mu H_{x s}\right), \\
H_{x s} & =h_{x s}^{(i)}(x) \cos k_{y}^{n}(y+b / 2) \cos k_{z}^{l} z
\end{aligned}
$$

where $h_{x s}^{(i)}(x)$ is defined as follows:

$$
\begin{aligned}
h_{x s}^{(i)}= & \frac{1}{\mu_{i}}\left(\cos k_{x s}^{i}\left(a_{i}-x\right),-\frac{\mu_{i}}{k_{x s}^{i}} \sin k_{x s}^{i}\left(a_{i}-x\right)\right) \zeta_{s}^{(i)}, \\
\zeta_{s}^{(i \leq N-2)}= & \left(\prod_{j=i+1}^{N-1} T_{s}^{(j)}\right)\left(\begin{array}{c}
-\frac{\mu_{N}}{k_{x s}^{N}} \sin k_{x s}^{N} w_{N} \\
\cos k_{x s}^{N} w_{N}
\end{array}\right) \frac{k_{x s}^{(N)}}{\sin k_{x s}^{(N)} a_{N}}, \\
\zeta_{s}^{(N-1)} & =\left(\begin{array}{c}
-\frac{\mu_{N}}{k_{x s}^{N}} \sin k_{x s}^{N} w_{N} \\
\cos k_{x s}^{N} w_{N}
\end{array}\right) \frac{k_{x s}^{(N)}}{\sin k_{x s}^{(N)} a_{N}}, \\
\zeta_{s}^{(N)} & =\left(\begin{array}{c}
0 \\
1
\end{array}\right) \frac{k_{x s}^{(N)}}{\sin k_{x s}^{(N)} a_{N}}, \\
T^{(i)} & \equiv\left(\begin{array}{cc}
\cos k_{x}^{i} w_{i} & -\frac{\mu_{i}}{k_{x}^{i}} \sin k_{x}^{i} w_{i} \\
\frac{k_{x}^{i}}{\mu_{i}} \sin k_{x}^{i} w_{i} & \cos k_{x}^{i} w_{i}
\end{array}\right) .
\end{aligned}
$$

The eigenfrequencies $\omega_{s}$ of the resonator are determined from the dispersion equations for the LSM and LSE waves $[15,19]$.

The dispersion equation of the LSM waves is [15]

$$
\left(\frac{k_{x}^{1}}{\varepsilon_{1}} \sin k_{x}^{1} w_{1}, \cos k_{x}^{1} w_{1}\right)\left(\prod_{i=2}^{N \geq 3} S^{(i)}\right)\left(\begin{array}{c}
\cos k_{x}^{N} w_{N} \\
\frac{k_{x}^{N}}{\varepsilon_{N}} \sin k_{x}^{N} w_{N}
\end{array}\right)=0
$$


and for the LSE waves [19] we have

$$
\begin{gathered}
\left(\cos k_{x}^{1} w_{1},-\frac{\mu_{1}}{k_{x}^{1}} \sin k_{x}^{1} w_{1}\right)\left(\prod_{i=2}^{N \geq 3} T^{(i)}\right) \\
\times\left(\begin{array}{c}
-\frac{\mu_{N}}{k_{x}^{N}} \sin k_{x}^{N} w_{N} \\
\cos k_{x}^{N} w_{N}
\end{array}\right)=0 .
\end{gathered}
$$

We now write down the electromagnetic-field norm $N_{s}$, introduced in expressions (23) through the use of the eigenfunctions $e_{x s}^{(i)}(x)$ and $h_{x s}^{(i)}(x)$.

For the LSM wave it is more convenient to define the norm using the magnetic field components. Substituting $H_{y s}$ and $H_{z s}$ from Eq. (26) into the second definition of the norm, we obtain

$$
\begin{aligned}
N_{s}^{(\mathrm{LSM})} & =\frac{1}{4 \pi} \int_{V_{R}} d V \mu\left[H_{y s} H_{y s}^{*}+H_{z s} H_{z s}^{*}\right] \\
& =\frac{k_{s}^{2} b L}{16 \pi \kappa_{n l}^{2}} \sum_{i=1}^{N} \varepsilon_{i}^{2} \mu_{i} \int_{a_{i-1}}^{a_{i}} d x\left(e_{x s}^{(i)}\right)^{2} .
\end{aligned}
$$

The norm of the LSM wave (34) is defined with the use of the electric field component perpendicular to the dielectric slabs.

For the LSE wave it is more convenient to define the norm using the electric field components. Substituting $E_{y s}$ and $E_{z s}$ from Eq. (29) into the first definition of the norm, we obtain

$$
\begin{aligned}
N_{s}^{(\mathrm{LSE})} & =\frac{1}{4 \pi} \int_{V_{R}} d V \varepsilon\left[E_{y s} E_{y s}^{*}+E_{z s} E_{z s}^{*}\right] \\
& =\frac{k_{s}^{2} b L \delta_{l, 0}}{16 \pi \kappa_{n l}^{2}} \sum_{i=1}^{N} \varepsilon_{i} \mu_{i}^{2} \int_{a_{i-1}}^{a_{i}} d x\left(h_{x s}^{(i)}\right)^{2},
\end{aligned}
$$

where $\delta_{l, 0}=2$ if $l=0$, and $\delta_{l, 0}=1$ if $l \neq 0$. As follows from (35), the LSE wave norm is defined with the use of the magnetic field component perpendicular to the dielectric slabs.

The expressions obtained for the potential (19), the amplitudes of expansion of the solenoidal field (24)-(26) and (29), and also the relativistic equations of bunch particle motion (6), enable us to analyze a self-consistent dynamics of excitation of the multilayered dielectric resonator by electron bunches.

\section{NUMERICAL INVESTIGATION}

For the numerical analysis of wakefield excitation, we will choose a five-zone dielectric resonator, a section of which is shown in Fig. 2. It represents the section of a rectangular five-zone dielectric waveguide having the length $L$, the ends of which are closed by metal walls transparent for charged particles. Two zones out of five are the vacuum zones, these are the so-called drive and accelerating channels. Electron bunches are injected into the drive channel. The charge of each bunch is equal to

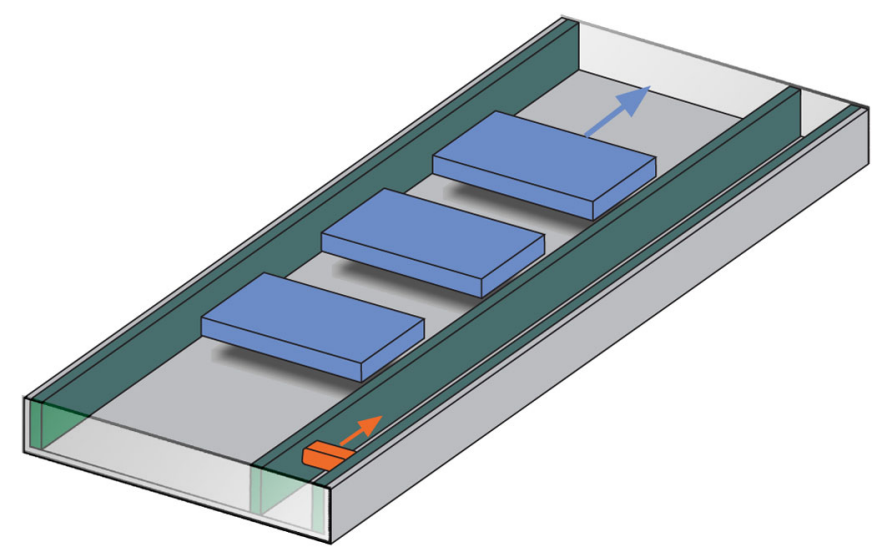

FIG. 2. Sectional view of the five-zone dielectric resonator. Drive bunches (blue) travel in a wide channel and the accelerated bunch (red) travels in a narrow channel. The arrows show the travel direction of drive and accelerated bunches. The end walls of the resonator are colored transparent light gray.

$50 \mathrm{nC}$, the electron bunch energy is $14 \mathrm{MeV}$. It is supposed that the bunches are rectangular in shape with homogeneous distribution of charge density. The bunch sizes are $L_{b x}=0.6 \mathrm{~cm}, L_{b y}=0.2 \mathrm{~cm}$, and $L_{b z}=0.4 \mathrm{~cm}$.

The main goal of numerical investigation of wakefield excitation by electron bunches in a multilayered resonator accelerating structure was to analyze the longitudinal boundedness effects in comparison with those investigated before [10] in a proper waveguide formulation. The cross-section dimensions of the resonator under study were: resonator width $a=1.8576 \mathrm{~cm}$, resonator height $b=0.6 \mathrm{~cm}$; thickness of dielectric slabs $w_{1}=$ $0.1237 \mathrm{~cm}, w_{3}=0.2288 \mathrm{~cm}, w_{5}=0.1051 \mathrm{~cm}$; thickness of drive channel $w_{4}=1.2 \mathrm{~cm}$, thickness of accelerating channel $w_{2}=0.2 \mathrm{~cm}$, permittivity of slabs $\varepsilon=4.76$, permeability of slabs $\mu=1$. The resonator length $L$ has been chosen such that it should be equal to ten wavelengths of the $\mathrm{LSM}_{3,1,20}$ mode resonant with a bunch (with symmetric transverse distribution in both vacuum channels) and was $L=9.988 \mathrm{~cm}$. This choice of the resonator parameters provided fulfillment of the conditions of the resonator wakefield accelerator concept [13].

Figure 3 shows longitudinal distributions of the axial electric field along the center of the drive channel in the waveguide and in the resonator, both excited by a single bunch. The distributions of total longitudinal wakefields along the center of drive and accelerating channels in the waveguide excited by a single charged bunch have been presented in papers $[1,10]$.

From Fig. 3 it follows that the field excited in the waveguide essentially differs from the field excited in the resonator. In the waveguide, the amplitudes of LSM and LSE fields are of the same order, whereas in the resonator the amplitudes of the LSE field (which also includes the potential field) essentially exceed the LSM field amplitude. The analysis of longitudinal and transverse amplitude 

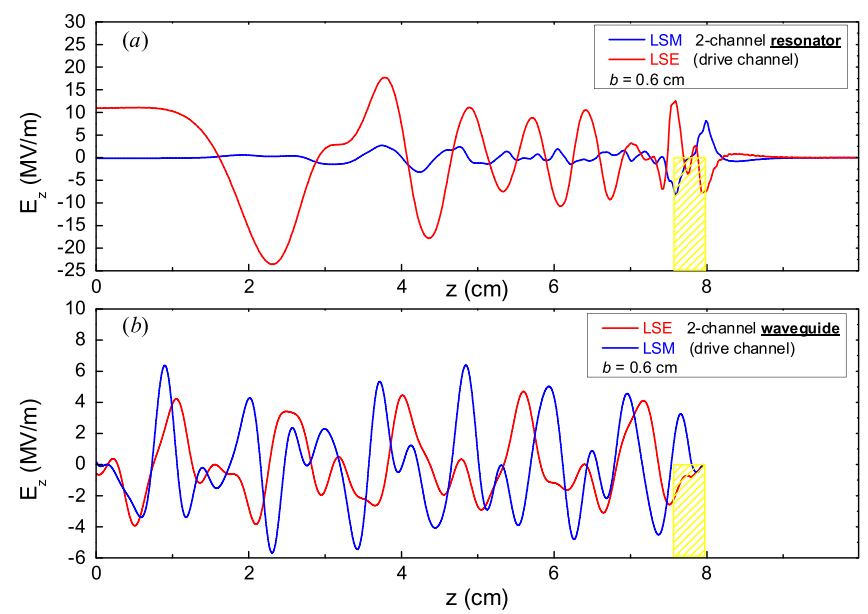

FIG. 3. Longitudinal profiles of an axial electric field excited by a single bunch along the center of the drive channel in the resonator (a), and a waveguide (b) at time $t=0.28 \mathrm{~ns}$ after bunch injection. The blue line is the LSM wave field, the red line is the LSE wave field; yellow rectangles show schematically the bunch positions (as well as in the following figures). The head of drive bunch has traveled the distance from $z=0$ to $z=8 \mathrm{~cm}$. The structure height is $b=0.6 \mathrm{~cm}$. In calculations of wakefields excited by a single electron bunch in a two-channel waveguide analytical results of papers $[15,19]$ have been used.

profiles of the modes, which constitute the LSM and LSE waves in the resonator, has shown that at the given parameters of the resonator the bulk LSE modes, which are far from being in resonance with the bunch, are rather strongly excited. These modes are not considered in the waveguide formulation of the problem.

It should be noted that the total wakefield [the sum of LSM and LSE modes shown in Fig. 3(a)], calculated on the basis of the derived set of equations, shows good agreement with the total wakefield computed with the use of the CST Particle Studio [20].

Figure 4 shows longitudinal electric field distributions along the center of the accelerating channel. It can be seen that the difference between the longitudinal electric field amplitudes in the resonator and those in the waveguide is less, but it is all the same considerable at large distances from the drive bunch. This change in the field behavior is connected with the group front motion of excited waves [11].

As it has been pointed out above, for using the structure under discussion in the two-beam acceleration scheme it is necessary to excite it at the $\mathrm{LSM}_{3,1,20}$ mode with a low level of parasitic harmonics. A further numerical calculation has shown that it can be achieved at an optimum height of the resonator. By varying the resonator height it is possible to control the amplitudes of total field components. When changing the resonator height, we fixed the frequency of the mode $\mathrm{LSM}_{3,1,20}$ resonant with the bunch and the symmetric distribution of its longitudinal field in the both vacuum channels. This was attained by matching the width

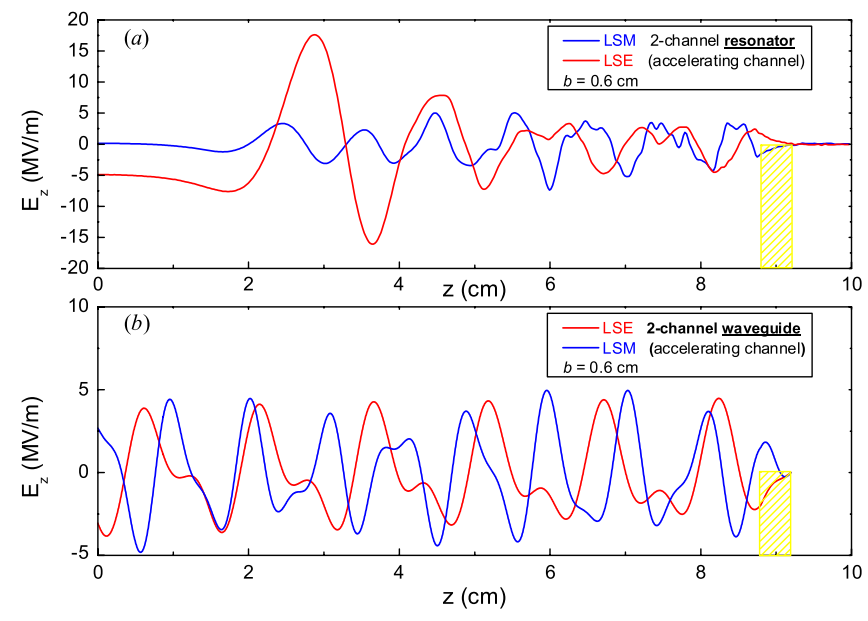

FIG. 4. Profiles of the axial electric field excited by a single bunch along the center of the accelerating channel in the resonator (a) and the waveguide (b) at time $t=0.31 \mathrm{~ns}$ after bunch injection. The blue line is the LSM wave field, the red line is the LSE wave field. The head of the drive bunch has traveled the distance from $z=0$ to $z=9 \mathrm{~cm}$. Structure height $b=0.6 \mathrm{~cm}$.

of dielectric slabs while keeping the same the width of drive and accelerating channels.

Figure 5 shows the longitudinal electric field profiles along the axis of the drive channel of the resonator excited by a single bunch, calculated for the resonator heights $b=1.2 \mathrm{~cm}$ [Fig. 5(a)] and $b=2.0 \mathrm{~cm}$ [Fig. 5(b)]. It is obvious from Fig. 5 that an increase in the accelerating structure height leads to an increase in the excited LSM field amplitude, and also to reduction in the LSE field amplitude, both in the region directly behind the bunch and in the region of group front. The reason why the ratio
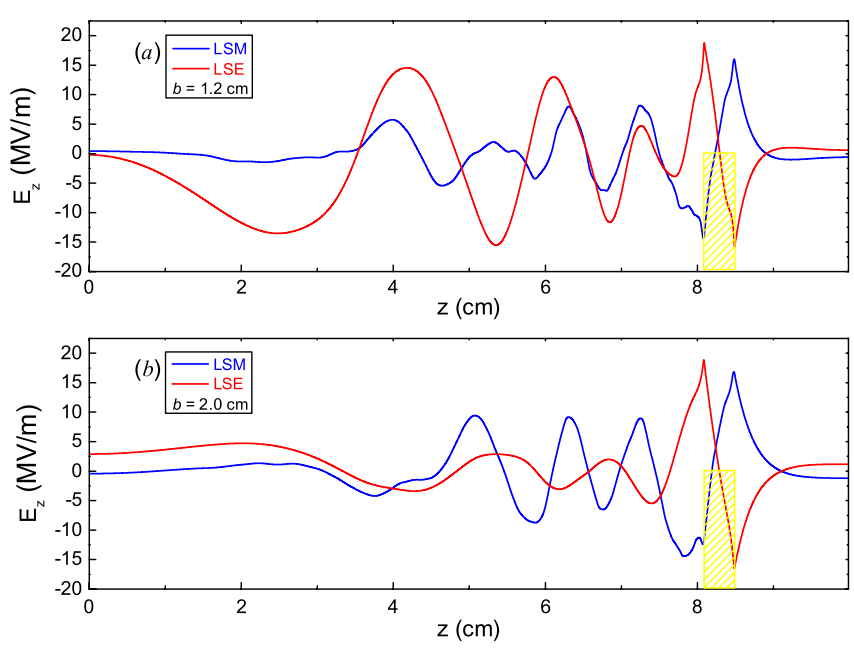

FIG. 5. Longitudinal distribution of axial electric field excited by a single bunch at the center of the drive channel of the resonator: (a) $b=1.2 \mathrm{~cm}$ and (b) $b=2.0 \mathrm{~cm}$. The blue line is the field of the LSM wave, the red line is the field of the LSE wave. 
of total field component amplitudes changes with an increasing resonator height lies in the change of the character of transverse distribution (surface or bulk) of LSM and LSE field mode amplitudes. With the height increase the contribution of low-frequency field modes also increases (that is proven true by the spectral analysis performed). It is necessary to note that for the accelerating channel a nonmonotonic dependence of the longitudinal electric field on the resonator height is observed. The maximum is reached at $b=1.2 \mathrm{~cm}$; with deviation from this value the amplitude of the longitudinal electric field decreases.

The calculated data on the resonator excitation by a sequence of 100 bunches for the resonator height $b=1.2 \mathrm{~cm}$ are presented in Fig. 6 . The bunch repetition rate was chosen to be equal to the frequency of $\mathrm{LSM}_{3,1,20}$ mode, and was $30 \mathrm{GHz}$. The distributions of the longitudinal electric field component correspond to the time point when the 100th bunch has completely entered the resonator.

Figure 6 demonstrates that a sequence of bunches regularizes the excited wakefield. Along with this, as the number of injected bunches increases, the period equal to the resonant wavelength (distance between the bunches) stands out in the longitudinal direction [Fig. 6(a)], while in the transverse direction [Fig. 6(b)] the form of the complete field becomes symmetric in the vacuum channels. The field amplitude at the center of the accelerating channel actually surpasses the field amplitude at the center of the drive channel.

Notice that the sequence of bunches of the same repetition rate does not suppress a great LSE field for the resonator height $b=0.6 \mathrm{~cm}$ (see Fig. 7). In this case, it is also impossible to attain the longitudinal field regularity and transverse field symmetry in the vacuum channels.
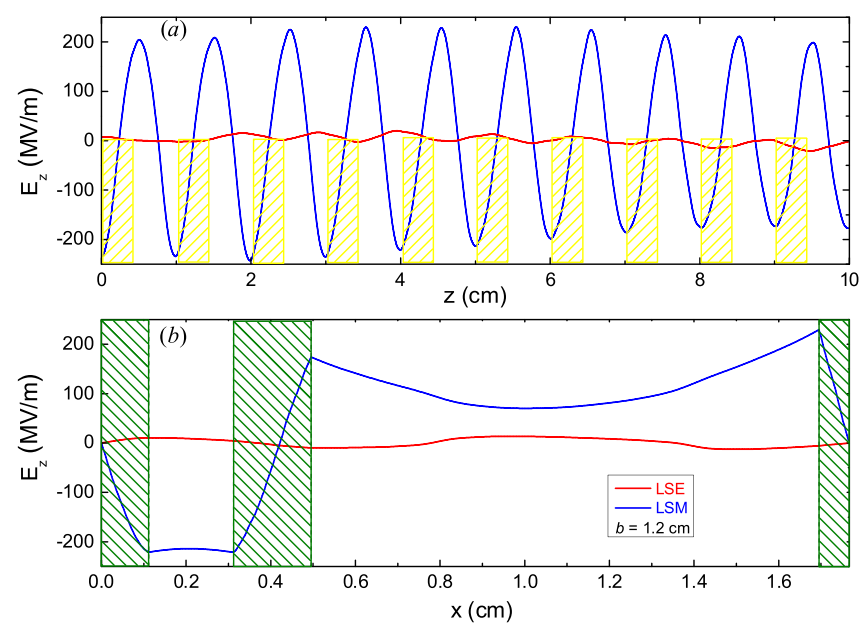

FIG. 6. Longitudinal (a) and cross-section (b) profiles of axial electric field excited along the center of the accelerating channel in the two-channel resonator by a sequence of 100 drive bunches. Structure height $b=1.2 \mathrm{~cm}$.

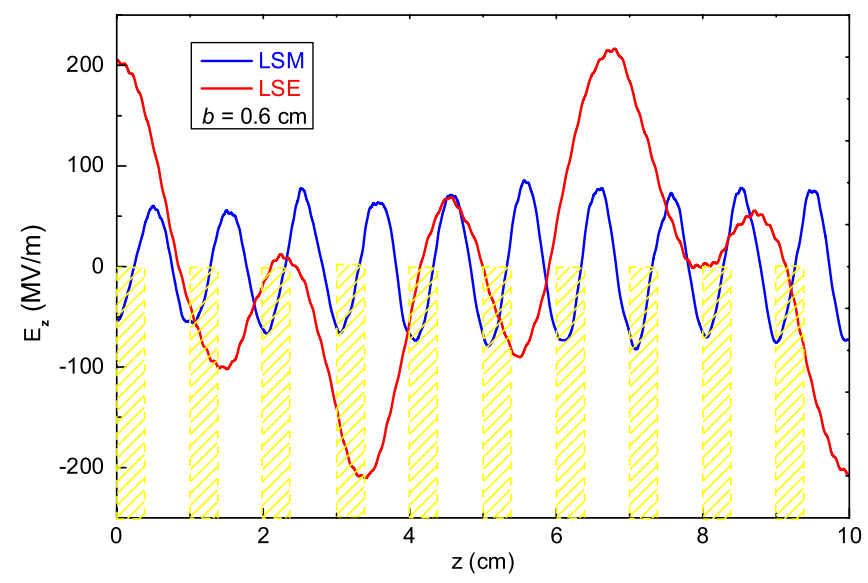

FIG. 7. Longitudinal profile of axial electric field excited along the center of the accelerating channel in the two-channel resonator by a sequence of 100 drive bunches. Resonator height $b=0.6 \mathrm{~cm}$.

\section{CONCLUSIONS}

The main results obtained in the studies are as follows.

The obtained set of nonlinear equations describes a selfconsistent dynamics of relativistic electron bunches in a multilayered dielectric resonator.

The dynamics of bunches is described by the equations of macroparticle motion, where the electromagnetic fields are set by superposition of Green's functions, in which the sources are the moving particles. Analytical solutions for the excited fields are represented as a superposition of the potential field and the solenoidal field. For the potential field, eigenfunctions and the condition of their orthogonality have been found; the equation for determination of eigenvalues (wave numbers, normal to dielectric layers) has been derived. The solenoidal field has been presented in the form of expansion in eigenfunctions of the LSM and LSE waves. Expressions for the amplitudes of these waves have been obtained. The eigenfunctions and the equations for eigenfrequencies of LSM and LSE waves, derived before for the waveguide formulation of structure excitation, have been generalized to the resonator case. Using the derived eigenfunctions, compact forms of the norms of these waves in the multilayered dielectric resonator have been found.

The undertaken numerical analysis of five-zone dielectric resonator excitation by electron bunches has demonstrated a vital role played by the longitudinal boundedness of the accelerator structure. The results of numerical calculations performed in the case of the two-channel resonator problem, where the effects of group velocity are considered properly, may differ considerably from the results of numerical calculations made in the waveguide formulation of the same problem. Previously, these effects were considered completely by numerical methods only, in PIC modeling. 
Numerical calculations using the set of equations obtained here show that a change in the height of the two-channel resonator leads to a change in the ratio of amplitudes of the total field components excited by the electron bunch.

Using a bunch train, it is possible to obtain an axial field of a rather high amplitude and regular in the longitudinal direction, with symmetric transverse distribution in vacuum channels.

\section{ACKNOWLEDGMENTS}

The authors are grateful to J.L. Hirshfield (Yale University, New Haven, and Omega-P, Inc.) and T.C. Marshall (Columbia University, New York) for their interest in the two-channel resonator formulation of wakefield excitation and the possibility of using their previous results of five-zone dielectric structure studies. This research was supported by the U.S. Department of Energy, Office of High Energy Physics, Advanced Accelerator R \& D.

[1] Wei Gai, in Proceedings of the Advanced Accelerator Concepts: 13 Workshop, edited by Carl B. Schroeder, Wim Leemans, and Eric Esarey, AIP Conf. Proc. No. 1086 (AIP, New York, 2009), pp. 3-11.

[2] Eric R. Colby, 35th International Conference on High Energy Physics, Paris, France, 2010 [http://indico.cern .ch/getFile.py/access?contribId=1033\&sessionId=57\&resId $=0 \&$ materialld $=$ slides \& confId $=73513]$.

[3] Wei Gai, Manoel Conde, John Gorham Power, and Chunguang Jing, in Proceedings of the 1st International Particle Acceleration Conference (IPAC'10 OC/ACFA, Kyoto, Japan, 2010), pp. 3428-3430.

[4] Wei Gai, M.E. Conde, R. Konecny, J. G. Power, P. Schoessow, X. Sun, and P. Zou, in Proceedings of the Advanced Accelerator Concepts: 9 Workshop, edited by Patric L. Colestock and Sandra Kelly, AIP Conf. Proc. No. 569 (AIP, Santa Fe, New Mexico, 2000), pp. 287-293.

[5] K. L.F. Bane, P. Chen, and P. B. Wilson, IEEE Trans. Nucl. Sci. 32, 3524 (1985).

[6] G. A. Voss and T. Weiland, DESY Report No. M-62-10, 1982.

[7] K. L. F. Bane, P. B. Wilson, and T. Weiland, in Physics of High Energy Particle Accelerator, AIP Conf. Proc. No. 127 (AIP, New York, 1985), pp. 875-928.
[8] C. Jing, A. Kanareykin, J. G. Power, M. Conde, Z. Yusof, P. Schoessow, and W. Gai, Phys. Rev. Lett. 98, 144801 (2007).

[9] C. Wang, T.C. Marshall, V.P. Yakovlev, and J. L. Hirshfield, in Proceedings of the 2005 Particle Acceleration Conference (IEEE, Knoxville, 2005), pp. 1333-1335.

[10] G. V. Sotnikov, T.C. Marshall, S. Y. Shchelkunov, A. Didenko, and J.L. Hirshfield, in Proceedings of the Advanced Accelerator Concepts: 13 Workshop, edited by Carl B. Schroeder, Wim Leemans, and Eric Esarey, AIP Conf. Proc. No. 1086 (AIP, New York, 2009), pp. 415-420.

[11] I. N. Onishchenko, D. Yu. Sidorenko, and G. V. Sotnikov, Phys. Rev. E 65, 066501 (2002).

[12] G. V. Sotnikov, T. C. Marshall, J. L. Hirshfield, and S. V. Shchelkunov, in Proceedings of the 7th International Workshop "Strong Microwaves: Sources and Applications", edited by Alexander G. Litvak [Inst. Appl. Phys. Russ. Acad. Sci. 1, 243 (2009)].

[13] I. N. Onishchenko and G. V. Sotnikov, Tech. Phys. 53, 1344 (2008).

[14] A short form of the theory has been presented in the report [21], where we gave the statement of the problem and the final set of equations. However, in that report there is no detailed procedure of deriving the set of equations and no example of numerical solution of the obtained equations is given. In the present paper, we describe the results of numerical simulations of the set of equations derived in Sec. III and carry out optimization of the geometrical dimensions of a multizone dielectric resonator in order to increase the accelerating gradient. Also, we compare the wakefields excited in the dielectric resonator with those excited in the dielectric waveguide.

[15] C. Wang and J. L. Hirshfield, Phys. Rev. ST Accel. Beams 9, 031301 (2006).

[16] L. A. Vainstein, Electromagnetic Waves (Radio i Sviaz, Moscow, 1988) (in Russian).

[17] Yu. V. Egorov, Partially Filled Rectangular Waveguides (Soviet. Radio, Moscow, 1967) (in Russian).

[18] L. Pincherle, Phys. Rev. 66, 118 (1944).

[19] G. V. Sotnikov, I. N. Onishchenko, J. L. Hirshfield, and T. C. Marshall, Probl. At. Sci. Technol., Ser. Nucl. Phys. Invest. 49, 148 (2008) [http://vant.kipt.kharkov.ua/ TABFRAME2.html].

[20] http://www.cst.com/Content/Products/PS/Overview.aspx.

[21] G. V. Sotnikov, K. V. Galaydych, and A. M. Naboka, in Proceedings of the 1st International Particle Acceleration Conference (Ref. [3]), pp. 4422-4424. 